\title{
Ecchymoses: an unusual manifestation of toxocariasis in children
}

\author{
V Pujitha Wickramasinghe', Sanath P Lamabadusuriya ${ }^{2}$ and Manel de S Wijesundera ${ }^{3}$ \\ (Index words: Platelet function tests, Toxocara canis antibodies, albendazole/thiabendazole)
}

\begin{abstract}
Objective To investigate the aetiology of multiple ecchymoses associated with eosinophilia.

Setting Professorial Paediatric Unit (PPU) at Lady Ridgeway Hospital (LRH) for children, Colombo.

Design Prospective study from July 1998 to April 2000.

Materials and methods Patients admitted to the PPU with multiple ecchymoses associated with an absolute eosinophilia (>1x1071) were investigated further to determine the possible aetiology; bleeding time, clotting time, platelet count, platelet function tests, stools examination and toxocara antibody tests were performed. Patients who were positive for toxocara were treated either with thiabendazole or albendazole. Patients who had geohelminth infestations were treated with mebendazole.
\end{abstract}

Results 14 children (11 boys) were studied. 7 were between 1 and 5 years, and 7 were above 5 years of age. The mean eosinophil count was $4.3 \times 1071$.

All patients had a prolonged bleeding time, but clotting time and platelet counts (mean value 220x1071) were normal. Platelet function tests were done in 5 patients. Twelve patients were positive for toxocariasis and the other 2 were positive for ascariasis.

All children who were positive for toxocariasis had contact with pet cats or dogs. Six patients were treated with thiabendazole and 6 with albendazole. The two patients who had ascariasis were treated with mebendazole. Posttreatment mean eosinophil count was $0.63 \times 1071$, and the bleeding time was 4 minutes.

Conclusion Toxocariasis could present with ecchymoses due to an acquired bleeding disorder. Awareness of this would help to investigate, diagnose and treat early, and lessen parental anxiety.

\section{Introduction}

Ecchymoses usually occur with thrombocytopaenia and commonly would be the presenting symptom of immune thrombocytopaenic purpura or bone marrow infiltration. A similar clinical presentation could also occur with platelet function defects. Abnormalities of platelet function can lead to a generalised haemorrhagic diathesis with normal numbers of platelets. Congenital platelet dysfunction is rare; acquired platelet dysfunction is more common, and usually associated with other conditions or due to medications such as aspirin (1). We came across a group of children who had ecchymoses with normal platelet counts and eosinophilia. There could be many causes for eosinophilia in our children, such as filariasis and intestinal helminthiasis. With a high stray dog and cat population one would also expect to see a high prevalence of visceral larva migrans (VLM) due to Toxocara canis and Toxocara cati. The object of this study was to identify the aetiology of the eosinophilia in children who had also ecchymoses and a normal platelet count.

\section{Materials and methods}

Children who were admitted to the PPU with a clinical presentation of multiple ecchymoses and an absolute eosinophilia (>1x 1071) were investigated to determine the aetiology, with bleeding time, clotting time, platelet count and platelet function tests. Stools were examined for evidence of geohelminth infestation. Toxocara antibody tests in the serum were performed at the Department of Parasitology, University of Peradeniya by an ELISA test based on detection of antibodies to excretory and secretory antigens (ESA) extracted from Toxocara canis L2 larvae cultured in vitro. This test has a $78 \%$ sensitivity and $92 \%$ specificity in VLM (2). Host-antibody responses to toxocara ESA antigens become detectable in 4 days to 4 weeks after infection. The results are expressed as an optic density (OD) value (Table 1). Patients with an OD values more than 0.2 were considered as positive for toxocariasis in the absence of a previous history of treatment for VLM.

Children who were positive for toxocara were treated either with thiabendazole SO $\mathrm{mg} / \mathrm{kg} / \mathrm{day}$ in two divided doses for 3 days or with albendazole $25 \mathrm{mg} / \mathrm{kg} /$ day in two divided doses for 3 days. Children who had ascariasis were treated with mebendazole $100 \mathrm{mg}$ bd for 3 days.

\section{Results}

Fourteen children (11 boys) presenting with ecchymoses and absolute eosinophilia were investigated. The mean eosinophil count was $4.3 \times 1071$ (Table 2).

They all had prolonged bleeding time (mean $9 \mathrm{~min}$ ), but clotting time and platelet counts (mean 220x1071) were normal. Platelet function tests were done in 5 patients and all showed normal aggregation with ristocetin $0.8 \mathrm{mg} / \mathrm{ml}$ and ADP Ifig. Platelet aggregation to collagen $4 \mathrm{jig}$, ristocetin. $1.2 \mathrm{mg} / \mathrm{ml}$, ADP 10 /i-g and arachidonic acid

Lecturer in Paediatrics, ${ }^{2}$ Senior Professor of Paediatrics, Faculty of Medicine, University of Colombo; and ${ }^{3}$ Professor of Parasitology, Faculty of Medicine, University of Peradeniya (Revised version accepted 7 September 2001. Corresponding author VPW) 
$2 \mathrm{mM}$ were abnormal in $80 \%, 40 \%, 60 \%$ and $60 \%$ respectively. Twelve children were positive for toxocariasis and the other two were positive for ascariasis. Mean value of toxocara serology OD was 0.87 (normal <0.2) before treatment (Table 1).

AH children directly or indirectly had contact with pet cats or dogs. After treatment the eosinophil counts and bleeding time came down dramatically. The mean eosinphil count had fallen to $0.63 \times 10^{\%} / 1$ and bleeding time to 4 minutes. The toxocara ELISA was repeated 6 months after treatment and the mean OD value was 0.47 . In all patients there was a decline in the toxocara ELISA OD value but still there were 5 patients with a value of over 0.7 , which indicates that it takes a long time to return to normal values. (Table 1).

\section{Table 1. Interpretation of ELISA OD values and the distribution among the patients}

Value and the interpretation Pretreatment Post-treatment
$<0.2$
$\begin{gathered}\text { (No serological evidence } \\ \text { of toxocariasis) }\end{gathered}$
$\quad 0.2$ to 0.7
$\begin{gathered}\text { (Compatible with past } \\ \text { infection or current } \\ \text { light infection) }\end{gathered}$
$\quad 4$
$>0.7$
(Compatible with
recent infection)

Table 2. Distribution of eosinophil count in the children before and after treatment

$\begin{array}{ccc}\text { Eosinophil count }(x l C P / 1) & \text { Pretreatment } & \text { Post-treatment } \\ <1 & - & 13 \\ 1-2.5 & 7 & 1 \\ 2.5-5 & 3 & \\ >5 & 4 & 0.31-2.8 \\ \text { Range } & 1.36-18.9 & 0.47\end{array}$

\section{Discussion}

Ecchymoses often detected on the legs of active healthy children are usually due to trauma. Unexplained spontaneous bruising usually indicates the presence of a bleeding diathesis and causes anxiety to parents. Acquired abnormalities of platelet function as assessed by in vitro studies or with a prolonged bleeding time could be due to a number of drugs, some food items, and spices and sys- temic conditions such as chronic renal failure and haematological disorders (3). The pathophysiology of acquired platelet dysfunction due to eosinophilia is not well understood, but this condition is frequently encountered in clinical practice. This non-thrombocytopaenic purpura with eosinophilia is caused by a transient acquired platelet function defect. This syndrome was first recognised in 1975 (4). All patients in our series were apparently healthy and did not have a past or family history of bleeding disorders, or of receiving any drugs. The only clinical manifestation was unexplained widespread ecchymoses. The abnormality found in the coagulation screening was prolonged bleeding time despite a normal platelet count. The platelet function tests, although done in only 5 patients, showed variable results, but the aggregation of platelets to collagen was abnormal in $80 \%$. Similar results were shown in a previous study (1). Though there was no uniformity in the results of the platelet function defects, the spontaneous recovery with return of bleeding time to normal following the treatment of the underlying cause of eosinophilia, suggests that the condition could have been an acquired defect of platelet function secondary to eosinophilia. Ecchymoses as a presenting symptom of VLM is not recognised. In this series a majority (85\%) of patients who had non-thrombocytopaenic purpura with eosinophilia were due to VLM. Therefore VLM should be considered as a strong possibility in children who present with ecchymoses, a normal platelet count and eosinophilia.

\section{Conclusion}

Toxocariasis could present with ecchymoses due to an acquired bleeding disorder associated with eosinophilia and platelet dysfunction. Awareness of this would help to investigate, diagnose and treat early and lessen parental anxiety.

\section{Acknowledgements}

We thank Dr A De Tissera of the MRI for performing the platelet function tests, and Mr R L A R Ranaweera, Department of Parasitology, Faculty of Medicine, Peradeniya, for performing the ELISA tests.

\section{References}

1. Suvatte V, Mahasandana C, Tanphaichitr V, TuchindaS. Acquired platelet dysfuction with eosinophilia: study of platelet function in 62 patients. Southeast Asian Journal of Tropical Medicine and Public Health 1979; 10: 358-67.

2. Glickman L, Schantz P, Dombroske R, Cypess R. Evaluation of serodiagnostic tests for visceral larva migrans. American Journal of Tropical Medicine and Hygiene 1978; 27: 492-8.

3. George JN, Shatth SS. The clinical importance of acquired abnormalities of platelet function. New England Journal of Medicine 1991; 324: 27-39.

4. Mitrakul C. Transient, spontaneous bruising with long bleeding time and normal platelet count. American Journal of Clinical Pathology 1975; 63: 81-6. 